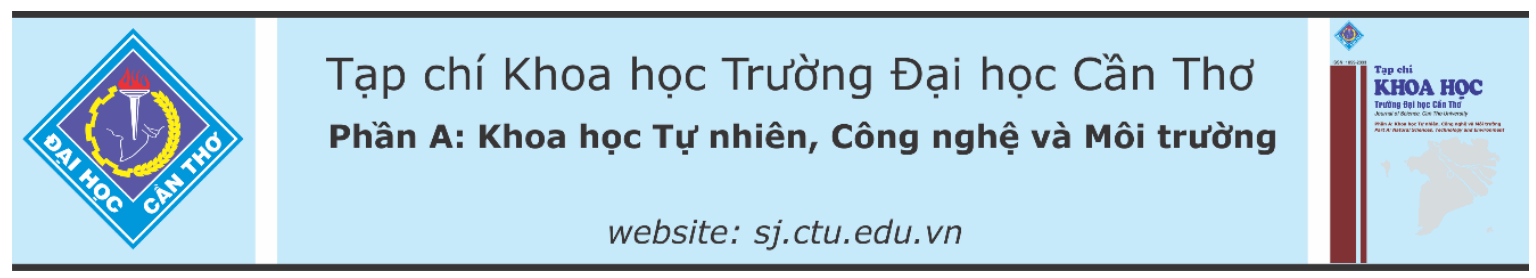

DOI:10.22144/ctu.jvn.2021.005

\title{
VÔ HỨ̛́NG HÓA PHI TUYẾN CHO BÀI TOÁN TỐI UUU ĐA MỤC TIÊU VỚI YẾU TỐ KHÔNG CHẮC CHẮN
}

\author{
Lâm Quốc $A n h^{1 *}$, Trần Quốc Duy ${ }^{2}$ và Trần Thị Tuyết Mai ${ }^{3}$ \\ ${ }^{1}$ Bộ môn Toán, Khoa Su phạm, Trương Đại học Cần Tho \\ ${ }^{2}$ Bộ môn Toán, Khoa Khoa học Cơ bản, Truờng Đại học Kỹ thuật - Công nghệ Cần Tho \\ ${ }^{3}$ Bộ môn Toán, Khoa Co bản, Truờng Đại học Tây Đô \\ *Người chịu trách nhiệm về bài viết: Lâm Quốc Anh (email: quocanh@ctu.edu.vn)
}

\section{Thông tin chung:}

Ngày nhận bài: 08/09/2020

Ngày nhận bài sưa: 13/10/2020

Ngày duyệt đăng: 27/03/2021

Title:

Nonlinear scalarization for multi-objective optimization problems under uncertainty

\section{Tù khóa:}

Bài toán tối ưu đa muc tiêu, hàm Gerstewitz, quan hệ thư tư tập, tối uu không chắc chắn, vô hướng hóa phi tuyến

\section{Keywords:}

Gerstewitz function, multiobjective optimization problem, nonlinear scalarization, set order relation, uncertain optimization

\begin{abstract}
In this paper, we study characterizations of minimal solutions to multiobjective optimization problems under with data containing the uncertainties defined in a given set. Firstly, we recall preliminaries of topological vector space ordered by a cone along with concepts related to the closedness, boundedness and properness properties of sets. Then, we consider properties of the nonlinear scalar function Gerstewitz in the topological vector space ordered by a solid cone and its generalizations. Finally, we introduce a concept of minimal solutions to the considered problems, and then based on properties of the generalization nonlinear scalar function, we establish characterizations of the minimal solutions. Besides, we give many examples to illustrate general concepts and properties to make the article easier to read.
\end{abstract}

\section{TÓM TẮT}

Trong bài báo này, chúng tôi nghiên cứu các đặc trung của nghiệm tối tiểu đối với bài toán tối ư đa muc tiêu với dũ liệu có chứa các yếu tố không chắc chắn được xác định trong một tập cho truớc. Cu thể, trước tiên, chúng tôi nhắc lại các kiến thức co bản của không gian vector topo được sắp thư tụ bởi nón nhu tính đóng, tính bị chặn và tính chính thuoòng của các tập. Sau đó, chúng tôi xem xét các tính chất của hàm vô hướng hóa phi tuyến dạng Gerstewitz trong không gian vector topo được sắp thư tư theo nón và các dạng mở rộng của nó. Cuối cùng, chúng tôi giới thiệu khái niệm điểm tối tiểu của bài toán đang xét và dưa vào các tính chất của hàm vô hướng phi tuyến suy rộng vì̀a đề xuất để thiết lập các đặc trung của nghiệm. Bên cạh đó, chúng tôi đưa ra các ví du để minh họa cho các khái niệm và tính chất tổng quát nhằm giúp cho bài viết dễ đọc hơn.

\section{GIỚI THIỆU}

Từ những năm 50 của thế kỷ trước, tối ưu hóa đã cho thấy được vai trò trong việc nghiên cứu nhiều vấn đề thực tế thuộc các lĩnh vực khác nhau, như vật lý, hóa học, sinh học, y học, kinh tế, kỹ thuật,... Một trong những khó khăn lớn nhất khi xem xét các tình huống trong thực tế của cuộc sống là các dữ liệu của bài toán thông thường sẽ không thể tránh khỏi việc chứa đựng những yểu tố không chắc chắn. Tính không chắc chắn của dữ liệu của các bài toán vừa nêu dường như là sự tất yếu, do dữ liệu của những bài toán này có thể là ngẫu nhiên hoặc do sai số, nó cũng có thể đến từ sai lầm của phép đo hoặc thiếu 
các tham số để đo lường, và đôi khi, tính không chắn chắn còn đến từ khả năng vật lý không thể hoàn thành chính xác các nhiệm vụ được yêu cầu trong bối cảnh thực tế của cuộc sống. Chính vì vậy, lớp các bài toán này ngày càng được nhiều nhà toán học quan tâm nghiên cứu, song hành với việc các chuyên gia mô hình hóa nghiên cứu phát triển các phương pháp, kỹ thuật điều tra đánh giá để có được số liệu chính xác đến mức có thể.

Hai trong số các phương pháp phổ biến nhất giải quyết các bài toán với dữ liệu chứa yếu tố không chắc chắn trong tối ưu hóa là tối ưu ngẫu nhiên và tối ưu vững. Đối với tối ưu ngẫu nhiên, các yếu tố không chắn chắn của dữ liệu các bài toán thường có thể được mô phỏng bằng các biến ngẫu nhiên có phân phối xác suất được xác định hoặc chúng có thể ước tính với độ chính xác đủ lớn. Để rõ thêm các chi tiết về tối ưu hóa ngẫu nhiên, bạn đọc có thể tìm hiểu ở một số công trình điển hình và quan trọng của lĩnh vực này (Popescu, 2007; Birge \& Louveaux, 2011). Trong khi đó, tối ưu vững dành để xem cho việc nghiên cứu các bài toán tối ưu với yếu tố không chẳn chắn của dữ liệu được giả định là không biết thông tin về phân phối xác suất chính xác của chúng, mà thay vào đó, các yếu tố không chắc chắn này được giả định rằng thuộc về tập hợp cho trước và thường được gọi là tập chứa yếu tố không chắc chắn của dữ liệu các mô hình đang xem xét. Để có thêm các thông tin về các mô hình tổng quát và các ứng dụng thực tế của các bài toán tối ưu vững, bạn đọc có thể xem các công trình (Ben-Tal et al., 2009; Kuhn et al., 2016; Doolittle et al., 2018; Jiao \& Lee, 2019). Một trong những phương pháp hữu hiệu được nhiều nhà toán học quan tâm trong việc nghiên cứu các bài toán tối ưu đa mục tiêu, tức là hàm mục tiêu có giá trị vector, là phương pháp vô hướng hóa. Trong đó, hàm vô hướng hóa dạng Gerstewitz là một trong những công cụ đã tỏ ra rất hữu dụng trong việc nghiên cứu tính chất nghiệm của nhiều lớp bài toán trong tối ưu hóa như bài toán tối ưu vector (Durea et al., 2017), bài toán tối ưu với hàm mục tiêu có giá trị tập (Khan et al., 2016), bài toán cân bằng (Tammer \& Zălinescu, 2014).

Lấy cảm hứng từ những công trình nghiên cứu đã đề cập ở trên, trong bài báo này chúng tôi xét bài toán tối ưu đa mục tiêu với dữ liệu có chứa các yếu tố không chắc chắn. Chúng tôi phát triển khái niệm hàm vô hướng hóa Gerstewitz và nghiên cứu các tính chất cơ bản của chúng. Dựa trên những khái niệm nghiệm hữu hiệu đã có trong tối ưu vector, chúng tôi đề xuất khái niệm nghiệm tối tiểu cho bài toán tối ưu đa mục tiêu với yếu tố không chắc chắn và sử dụng các tính chất của hàm vô hướng hóa
Gerstewitz mở rộng, chúng tôi đưa ra đặc trưng của nghiệm tối tiểu cho các bài toán đang xem xét.

\section{KIẾN THỨC CHUẨN BI!}

Trong bài báo này, chúng ta xét $Y$ là một không gian topo tuyến tính thực.

Định nghĩa 2.1 Tập $C \subset Y$ được gọi là tập lồi nếu với mỗi $x, y \in C$ và $\alpha \in[0,1]$ ta có $\alpha x+$ $(1-\alpha) y \in C$.

Định nghĩa 2.2 Tập $C \subset Y$ được gọi là nón nếu với mọi $x \in C$ và mọi $\alpha>0$ ta luôn có $\alpha x \in C$. Nón $C$ được gọi là nón có đỉnh nếu $C \cap(-C)=\left\{0_{Y}\right\}$. Nón $C$ được gọi là nón lồi nếu $C$ là nón và là tập lồi.

Ví dụ 2.1 Trong $\mathbb{R}$, ta chỉ có các nón $\mathbb{R}, \mathbb{R}_{+}, \mathbb{R}_{-},\{0\}$. Trong khi đó, đối với $\mathbb{R}^{2}$ ta có vô số các nón thứ tự, trong đó nón $\mathbb{R}_{+}^{2}$ là nón được nhiều người quan tâm bởi ý nghĩa thực tế của nó. gọi là

Định nghĩa 2.3 Một tập khác rỗng $A \subset Y$ được

$C$-đóng nếu $A+C$ là một tập đóng;

$C$-bị chặn nếu với mỗi lân cận $V$ của $0_{Y}$ tồn tại số $t>0$ sao cho $A \subset t V+C$;

$C$-chính thường nếu $A+C \neq Y$.

Ta ký hiệu $\wp(Y)$ là một họ các tập con không rỗng $C$-chính thường của $Y$ và $\mathcal{M}(\wp(Y), \mathbb{R})$ là tập hợp tất cả các hàm từ $\wp(Y)$ vào $\mathbb{R}$.

Ví dụ 2.2 Trong $\mathbb{R}$, ta có

Tập $A=[0,1)$ là $\mathbb{R}_{+}$-đóng vì $A+\mathbb{R}_{+}=[0,1)+$ $\mathbb{R}_{+}=[0,+\infty)$ là một tập đóng.

Tập $A=\left\{x^{2}-1: x \in \mathbb{R}, x \neq 0\right\}$ là $\mathbb{R}_{+}$-bị chặn vì $A$ là tập bị chặn dưới.

Với mỗi $A, B \in \wp(Y)$, ta xét quan hệ so sánh giữa các tập, ký hiệu bởi $\preccurlyeq$, như sau

$A \preccurlyeq B$ nếu và chỉ nếu $B \subset A+C$,

$A \prec B$ nếu và chỉ nếu $B \subset A+\operatorname{int} C$.

Ví dụ 2.3 Có 2 em học sinh $A n$ và Bình vừa tham dự kỳ thi tốt nghiệp THPT năm học 2020. Gọi $A$ và $B$ lần lượt là hai tập hợp điểm số các môn Toán, Văn, Anh Văn và môn Tổ hợp của hai em như sau: $A=\{9,8,6,10\}$ và $B=\{8,9,8.5,7\}$. Như vậy, trên cơ sở điểm số như trên ta có thể nói rằng $A \preccurlyeq B$ vì $B \subset A+\mathbb{R}_{+}$.

Định nghĩa 2.4 (Hernández \& Rodríguez-Marín, 2007) Trong $\wp(Y)$ ta xét quan hệ $\sim$ giữa các tập được xác định như sau $A \sim B \Leftrightarrow A \preccurlyeq B$ và $B \preccurlyeq A$. 
Khi đó, quan hệ là một quan hệ tương đương trên $\wp(Y)$. Lớp tương đương của tập $A \in \wp(Y)$ được ký hiệu bởi $[A]$. Bằng cách kiểm tra trực tiếp ta có

$$
B \in[A] \Leftrightarrow A+C=B+C .
$$

Định nghĩa 2.5 (Han, 2019) Cho $a \in Y$ và $e \in$ $-\operatorname{int} C$. Hàm Gerstewitz $\varphi_{e, a}: Y \rightarrow \mathbb{R}$ được định nghĩa như sau $Y$.

$$
\varphi_{e, a}(y)=\min \{t \in \mathbb{R}: y \in t e+a+C\}, \forall y \in
$$

Ví dụ 2.4 Trong $\mathbb{R}^{2}$, chọn $a=(0 ; 0) \in \mathbb{R}^{2}, C=$ $\mathbb{R}_{+}^{2}, e=(-1 ;-1) \in-\operatorname{int} \mathbb{R}_{+}^{2}$. Khi đó, với $y=$ $(-1 ; 2)$, ta có

$$
\begin{aligned}
\varphi_{e, a}(-1 ; 2) & =\min \{t \in \mathbb{R}:(-1 ; 2) \\
& \left.\in t(-1 ;-1)+(0 ; 0)+\mathbb{R}_{+}^{2}\right\} \\
= & \min \{t \in \mathbb{R}:(-1 ; 2) \\
& \left.\in(-t ;-t)+\mathbb{R}_{+}^{2}\right\} \\
= & \min \left\{t \in \mathbb{R}:(-1+t ; 2+t) \in \mathbb{R}_{+}^{2}\right\} \\
= & \min \{t \in \mathbb{R}:-1+t \geq 0 \text { và } 2+t \\
& \geq 0\} \\
= & \min \{t \in \mathbb{R}: t \geq 1 \text { và } t \geq-2\}=1 .
\end{aligned}
$$

Bổ đề 2.1 (Hernández \& Rodríguez-Marín, 2007) Cho $t \in \mathbb{R}, e \in-\operatorname{int} C$ và $y \in Y$. Khi đó,

$$
\begin{gathered}
\varphi_{e, a}(y)<t \Leftrightarrow y \in t e+a+\operatorname{int} C \\
\varphi_{e, a}(y) \leq t \Leftrightarrow y \in t e+a+C \\
\varphi_{e, a}(y)>t \Leftrightarrow y \notin t e+a+C .
\end{gathered}
$$

Nếu chúng ta thay thế $a$ bằng một tập con không rỗng $A \subset Y$, ta sẽ được hàm $\varphi_{e, A}: Y \rightarrow \mathbb{R} \cup$ $\{-\infty\}$ như sau

$\varphi_{e, A}(y)=\inf \{t \in \mathbb{R}: y \in t e+A+C\}, \forall y \in Y$.

Một cách tương đương, $\varphi_{e, A}(y)=\inf _{a \in A} \varphi_{e, a}(y)$ với mỗi $y \in Y$.

Tiếp theo, chúng ta sẽ mở rộng hàm Gerstewitz cho hai tập con khác rỗng của $Y$ như sau

$$
\begin{array}{r}
G_{e}(\cdot, \cdot): \wp(Y) \times \wp(Y) \rightarrow \mathbb{R} \cup\{\infty\} \\
(A, B) \quad \mapsto G_{e}(A, B)=\sup _{b \in B}\left\{\varphi_{e, A}(b)\right\} .
\end{array}
$$

Bổ đề 2.2 (Zhang et al., 2009) Cho $A$ là tập $C$ đóng và $B$ là tập $C$-bị chặn. Khi đó, ta có

$$
G_{e}(A, B)=\min \{t \in \mathbb{R}: B \subset t e+A+C\} .
$$

Bổ đề 2.3 (Zhang et al., 2009) Cho $t \in \mathbb{R}, A$ là tập $C$-đóng và $B$ là tập $C$-bị chặn. Khi đó, ta có

$$
G_{e}(A, B) \leq t \Leftrightarrow B \subset t e+A+C .
$$

Bổ đề 2.4 (Zhang et al., 2009) Giả sử $k_{0} \in \operatorname{int} C$, $A, A_{1}, A_{2}, B \in \wp(Y), t \in \mathbb{R}, A, A_{1}, A_{2}$ là tập $C$-đóng và $B$ là tập $C$-bị chặn. Khi đó,

$$
\begin{aligned}
& G_{e}\left(A+\varepsilon k_{0}, B\right)=G_{e}(A, B)+\varepsilon, \forall \varepsilon \geq 0 ; \\
& G_{e}\left(A, B+\varepsilon k_{0}\right)=G_{e}(A, B)-\varepsilon, \forall \varepsilon \geq 0 ; \\
& \text { Nếu } A_{1} \preccurlyeq A_{2} \text {, thì } G_{e}\left(A_{1}, B\right) \leq G_{e}\left(A_{2}, B\right) ; \\
& G_{e}(A, B)<t \Leftrightarrow B \subset t e+A+\operatorname{int} C .
\end{aligned}
$$

Bổ đề 2.5 (Hernández \& Rodríguez-Marín, 2007) Cho $A \in \wp(Y)$. Khi đó, hàm $G_{e}(\cdot, A)$ là hàm tăng trên $\wp(Y)$.

Bổ đề 2.6 (Hernández \& Rodríguez-Marín, 2007) Cho $A \in \wp(Y)$ là một tập $C$-đóng. Khi đó, những phát biểu sau đây là đúng

$$
\begin{aligned}
& \text { Nếu } A \in[B] \text { thì } G_{e}(A, B)=G_{e}(B, A)=0 . \\
& A \preccurlyeq B \text { khi và chỉ khi } G_{e}(A, B) \leq 0
\end{aligned}
$$

Định nghĩa 2.6 (Hernández \& Rodríguez-Marín, 2007) Cho họ các tập con $\mathcal{H} \subset \wp(Y)$. Một hàm số $T: \wp(Y) \rightarrow \mathbb{R} \cup\{ \pm \infty\}$ được gọi là tăng trên $\mathcal{H}$ nếu $A, B \in \mathcal{H}$ và $A \leqslant B$ ta suy ra $T(A) \leq T(B)$.

Ví dụ 2.5 Cho $Y=\mathbb{R}, C=\mathbb{R}_{+}$và $\mathcal{H}$ là họ các tập con compact trong $\mathbb{R}$. Xét hàm $T: \wp(\mathbb{R}) \rightarrow \mathbb{R} \cup$ $\{ \pm \infty\}$ được xác định bởi $T(A)=\inf A$. Khi đó, $T$ là tăng trên $\mathcal{H}$.

\section{NGHIẸM TỐI TIỂU CỦA BÀI TOÁN TỐI U'U HÓA ĐA MỤC TIÊU VỚI YẾU TỐ KHÔNG CHẮC CHẮN}

Cho $X, Y, Z$ là các không gian vector tô pô tuyến tính, $C \subset Y$ là nón lồi, đóng, có đỉnh và có phần trong khác rỗng. Bài toán tối ưu vector được xác định ở dạng

$$
\text { (P) } \begin{aligned}
\min & f(x) \\
\text { s.t. } & x \in K
\end{aligned}
$$

trong đó $f: X \rightarrow Y$ là một hàm mục tiêu và $K \subset$ $X$ là một tập chấp nhận được. Bây giờ, chúng ta giả sử thêm rằng hàm mục tiêu $f$ sẽ phụ thuộc vào yếu tố không chắc chắn $\xi$ được cho trong tập $u \subset$ $Z \backslash\{\varnothing\}$, và sẽ được gọi là tập điều kiện không chắc chắn. Như vậy, bài toán tối ưu đa mục tiêu không chắc chắn $P(\mathcal{U})$ được cho bởi một họ $\{P(\xi): \xi \in \mathcal{U}\}$ của các bài toán tối ưu ở dạng

$$
\begin{aligned}
& P(\xi) \quad \min \\
& \text { s.t. } \quad x \in K
\end{aligned}
$$

trong đó $f: X \times \mathcal{U} \rightarrow Y$ là một hàm mục tiêu và $K \subset X$ là một tập chấp nhận được. 
Xét hàm đa trị $F: X \rightrightarrows Y$ được xác định bởi $F(x)=f_{u}(x)=\{f(x, \xi): \xi \in \mathcal{U}\}$, với mỗi $x \in X$.

Định nghĩa 3.1 Cho bài toán tối ưu đa mục tiêu không chắc chắn $P(\mathcal{U})$. Điểm $\bar{x}$ được gọi là nghiệm tối tiểu của bài toán $P(\mathcal{U})$ nếu $f_{u}(x) \preccurlyeq f_{u}(\bar{x})$ với $x \in K$ ta suy ra $f_{u}(\bar{x}) \preccurlyeq f_{u}(x)$.

Ví dụ 3.1 Xét $X=\mathbb{R}, Y=Z=\mathbb{R}^{2}$, xét $C=$ $\mathbb{R}_{+}^{2}, \mathcal{U}=[0,1] \times[0,2 \pi]$. Hàm $f: \mathbb{R} \times \mathcal{U} \rightarrow \mathbb{R}^{2}$ xác định bởi $f(x, \xi)=\left(\xi_{1} \cos \xi_{2}+x ; \xi_{1} \sin \xi_{2}-x\right)$ với mỗi $x \in \mathbb{R}$ và $\xi=\left(\xi_{1}, \xi_{2}\right) \in \mathcal{U}$. Khi đó,

$$
\begin{gathered}
F(x)=\left\{f(x, \xi)=\left(\xi_{1} \cos \xi_{2}+x ; \xi_{1} \sin \xi_{2}-x\right): \xi\right. \\
\left.=\left(\xi_{1}, \xi_{2}\right) \in \mathcal{U}\right\} \\
=\left\{\left(y_{1}, y_{2}\right):\left(y_{1}-x\right)^{2}+\left(y_{2}+x\right)^{2} \leq 1\right\} .
\end{gathered}
$$

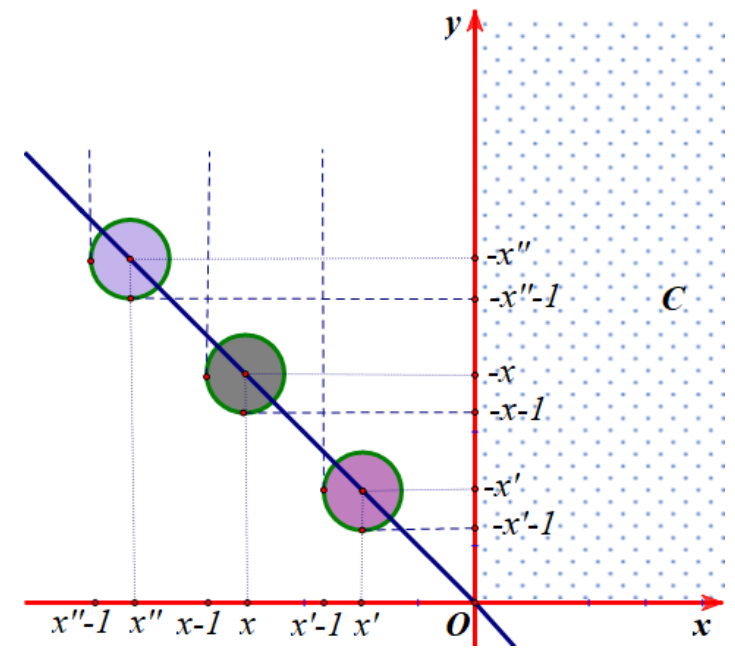

Bằng cách kiểm tra trực tiếp ta thấy rằng tập nghiệm tối tiểu của bài toán là tập $\mathbb{R}$.

Định lý 3.1 Cho $e \in-\operatorname{int} C$. Xét bài toán $P(\mathcal{U})$, giả sử rằng $U$ là tập compact, $f_{U}(x)$ có giá trị $C$-bị chặn, $C$-đóng và $C$-chính thường trên $X$. Khi đó, $\bar{x} \in$ $X$ là một nghiệm tối tiểu của bài toán $P(\mathcal{U})$ nếu và chỉ nếu hàm $G_{e}\left(\cdot, f_{u}(\bar{x})\right) \in M(\wp(Y), \mathbb{R})$ tăng trên $\wp(Y)$ và với mối $x \in X$ nếu $f_{u}(x) \in\left[f_{u}(\bar{x})\right]$ thì $G_{e}\left(f_{u}(x), f_{u}(\bar{x})\right)=0$; và nếu ngược lại thì $G_{e}\left(f_{u}(x), f_{u}(\bar{x})\right)>0$. Hơn nữa, với mọi $A \in \wp(Y)$ sao cho $A \preccurlyeq f_{u}(\bar{x})$ ta luôn có $G_{e}\left(A, f_{u}(\bar{x})\right) \leq 0$.

\section{Chứng minh}

$(\Longrightarrow)$ Giả sử $\bar{x}$ là một nghiệm tối tiểu của bài toán $P(\mathcal{U})$. Ta cố định bất kỳ giá trị $e \in-\operatorname{int} C$. Áp dụng Bồ đề 2.5 , ta suy ra hàm $G_{e}\left(\cdot, f_{u}(\bar{x})\right) \in M(\wp(Y), \mathbb{R})$ tăng trên $\wp(Y)$. Vì thế theo Bồ đề $2.6(\mathrm{a})$, chúng ta có nếu $x \in X, f_{u}(x) \subset f_{u}(\bar{x})+C$ và $f_{u}(\bar{x}) \subset$ $f_{u}(x)+C$ thì $G_{e}\left(f_{u}(x), f_{u}(\bar{x})\right)=0$.
Bởi vì $\bar{x}$ là một nghiệm tối tiểu của bài toán $P(\mathcal{U})$ với mỗi $x \in X$ sao cho $f_{u}(x) \notin\left[f_{u}(\bar{x})\right]$ ta có $f_{u}(x)$ * $f_{u}(\bar{x})$. Vì vậy từ Bổ đề $2.6(\mathrm{~b})$, ta được $G_{e}\left(f_{u}(x), f_{u}(\bar{x})\right)>0$. Từ $A \preccurlyeq f_{u}(\bar{x})$ ta có được $G_{e}\left(A, f_{u}(\bar{x})\right) \leq 0$.

$(\Longleftarrow)$ Ngược lại, nếu những điều kiện đúng cho hàm $G_{e}$ khi đó ta phải chứng minh rằng $\bar{x}$ là một nghiệm tối tiểu của bài toán $P(\mathcal{U})$.

Giả sử ngược lại, nếu $\bar{x}$ không là một nghiệm tối tiểu của bài toán $P(\mathcal{U})$ khi đó tồn tại $x^{\prime} \in X$ sao cho $f_{u}\left(x^{\prime}\right) \preccurlyeq f_{u}(\bar{x})$ nhưng $f_{u}(\bar{x}) \leqslant f_{u}\left(x^{\prime}\right)$, và do đó $f_{u}\left(x^{\prime}\right) \notin\left[f_{u}(\bar{x})\right]$. Theo giả thiết của điều kiện cần, ta có $G_{e}\left(f_{u}\left(x^{\prime}\right), f_{u}(\bar{x})\right)>0$.

Mặt khác, vì $f_{u}\left(x^{\prime}\right) \preccurlyeq f_{u}(\bar{x})$ ta có $G_{e}\left(f_{u}\left(x^{\prime}\right), f_{u}(\bar{x})\right) \leq 0$. Đây là điều vô lý và định lý được chứng minh.

\section{KẾT LUẬN}

Trong bài báo này, chúng tôi nghiên cứu điều kiện cho nghiệm tối tiểu của bài toán tối ưu đa mục tiêu với yếu tố không chắc chắn thông qua các đặc trưng của hàm vô hướng hóa phi tuyến dạng Gerstewitz. Cách tiếp cận và các kết quả đạt được là mới và khác với các kết quả đã có. Hơn nữa, chúng tôi cho rằng với những điều chỉnh thích hợp, phương pháp vô hướng hóa này cũng có thể dùng để xem xét điều kiện nghiệm cho các dạng nghiệm hữu hiệu khác trong tối ưu không chắc chắn.

\section{TÀI LIỆU THAM KHẢO}

Ben-Tal, A., Ghaoui, L.E., \& Nemirovski, A. (2009). Robust Optimization. Princeton University Press.

Birge, J.R., \& Louveaux, F. (2011). Introduction to Stochastic Programming. Springer.

Doolittle, E.K., Kerivin, H.L.M., \& Wiecek, M.M. (2018). Robust multiobjective optimization with application to internet routing. Annals of Operations Research, 271(2): 487-525.

Durea, M., Strugariu, R., \& Tammer, C. (2017). On some method to derive necessary and sufficient optimality conditions in vector optimization. Journal of Optimization Theory and Applications, 175(3): 738-763.

Han, Y. (2019). Nonlinear scalarizing functions in set optimization problems. Optimization. 68(9): 1685-1718.

Hernández, E., \& Rodríguez-Marín, L. (2007). Existence theorems for set optimization problems. Nonlinear Analysis: Theory, Methods and Applications. 67(6): 1726-1736. 
Jiao, L., \& Lee, J.H. (2019). Finding efficient solutions in robust multiple objective optimization with SOS-convex polynomial data. Annals of Operations Research, 1-18.

Khan, A.A., Tammer, C., \& Zălinescu, C. (2016). Set-valued Optimization. Springer.

Köbis, E., Tam, L.T., \& Tammer, C. (2018). A generalized scalarization method in set optimization with respect to variable domination structures. Vietnam Journal of Mathematics. 46(1): 95-125.
Kuhn, K., Raith, A., Schmidt, M., \& Schobel, A. (2016). Bi-objective robust optimisation. European Journal of Operational Research. 252(2): 418-431.

Popescu, I. (2007). Robust mean-covariance solutions for stochastic optimization. Operations Research, 55(1): 98-112.

Zhang, W.Y., Li, S.J., \& Teo, K.L. (2009). Wellposedness for set optimization problems. Nonlinear Analysis: Theory, Methods and Applications, 71(9): 3769-3778. 